Pacific Journal of Mathematic 


\title{
SUBFUNCTIONS AND THE DIRICHLET PROBLEM
}

\author{
LLOYD K. JACKSON
}

1. Introduction. In previous papers $[1 ; 6]$ the notion of subharmonic functions was generalized by replacing the dominating family of harmonic functions by a more general family of functions. The object was to require of the dominating functions the minimum properties necessary to study the boundary value problem by subfunction techniques. In a natural way these properties were separated into two parts: first, those properties sufficient to obtain functions which are solutions in the interior of a domain and, second, those properties sufficient to obtain agreement of the solution with the prescribed boundary values on the boundary of the domain. In particular the aim was to choose properties which would be sufficient to insure that a solution would take on prescribed boundary values at any boundary point $p$ at which an exterior circle could be drawn intersecting the closed domain only in the point $p$. In a recent paper Inoue [5] points out an error in this second aspect of [1]. Inoue then lists properties of the dominating functions which are sufficient to insure the regularity of boundary points at which exterior triangles can be drawn. In his paper these properties are embodied in six postulates the first four of which are essentially the same as the first four postulates of [1]. Postulates 5 and 6 given by Inoue are used in studying the behavior at the boundary and are naturally more restrictive but they are such that the theory can be applied to elliptic partial differential equations which have the property that the difference between two solutions is subharmonic when positive.

In the present paper we use only the portion of the theory of subfunctions which is based on the first four postulates of [1] to obtain some results concerning the Dirichlet problem for certain types of elliptic equations. We shall give some results concerning the linear equation

$$
\Delta z+a(x, y) z_{x}+b(x, y) z_{y}+c(x, y) z=f(x, y),
$$

where $\Delta z=\frac{\partial^{2} z}{\partial x^{2}}+\frac{\partial^{2} z}{\partial y^{2}}$, and the quasi-linear equation

$$
a(p, q) r+2 b(p, q) s+c(p, q) t=0,
$$

where $p=\frac{\partial z}{\partial x}, q=\frac{\partial z}{\partial y}, r=\frac{\partial^{2} z}{\partial x^{2}}, s=\frac{\partial^{2} z}{\partial x \partial y}$, and $t=\frac{\partial^{2} z}{\partial x^{2}}$. In particular we

Received September 9, 1957. In revised form January 6, 1958. 
shall give a theorem concerning the Dirichlet problem for the minimal surface equation

$$
\left(1+q^{2}\right) r-2 p q s+\left(1+p^{2}\right) t=0
$$

for non-convex regions. The result is quite weak but is perhaps of some interest since results ef this type are very meagre indeed.

2. $\{\boldsymbol{F}\}$-functions and sub- $\{\boldsymbol{F}\}$ functions. In this section we shall list for convenience the postulates satisfied by the $\{F\}$-functions and some theorems given in [1]. For simplicity our language will be in terms of the plane, however, our statements in this section could be phrased in terms of Euclidean space of any number of dimensions.

Let $D$ be a given plane domain and let $\{\kappa\}$ be the family of all circles with radii less than some fixed number and such that $\bar{K}=K+\kappa \subset D$ where $K$ is the open circle bounded by $\kappa$ and $\bar{K}$ its closure. Throughout the paper we shall use $\Omega$ to indicate an arbitrary bounded domain such that $\bar{\Omega} \subset D$ and the boundary of $\Omega$ will be represented by $\omega$. We shall use single small italic letters in this section to represent points in the plane.

Let there be given a family of functions $\{F(x)\}$, which we shall call $\{F\}$-functions, satisfying the postulates that follow.

Postulate 1. For any $\kappa \in\{\kappa\}$ and any continuous boundary value function $h(x)$ defined on $k$, there is a unique $F(x ; h ; k) \in\{F(x)\}$ such that

(a) $F(x ; h ; \kappa)=h(x)$ on $\kappa$,

and

(b) $F(x ; h ; \kappa)$ is continuous on $\bar{K}$.

Postulate 2. If $h_{1}(x)$ and $h_{2}(x)$ are continuous on $\kappa \in\{k\}$ and if $h_{1}(x)-h_{2}(x) \leqq M$ on $\kappa, M \geqq 0$, then

$$
F\left(x ; h_{1} ; \kappa\right)-F\left(x ; h_{2} ; \kappa\right) \leqq M
$$

in $K$; further, if the strict inequality holds at a point of $\kappa$, then the strict inequality holds throughout $K$.

Postulate 3. For any $\kappa \in\{\kappa\}$ and any collection $\left\{h_{\nu}(x)\right\}$ of functions $h_{\nu}(x)$ which are continuous and uniformly bounded on $\kappa$, the functions $F\left(x ; h_{\nu} ; k\right)$ are equicontinuous in $K$.

Definition 1. The function $s(x)$ is defined to be a sub- $\{F\}$ function, or simply a subfunction, in $D$ provided

(a) $s(x)$ is bounded on every closed subset of $D$,

(b) $s(x)$ is upper semicontinuous in $D$, 
and

(c) $s(x) \leqq F(x) \in\{F(x)\}$ on $\kappa \in\{\kappa\}$ implies $s(x) \leqq F(x)$ in $K$.

Definition 2. The function $S(x)$ is defined to be a super- $\{F\}$ function or a superfunction in $D$ provided $-S(x)$ is a sub-\{-F\} function in $D$.

Let $g(x)$ be a bounded function defined on $\omega$, the boundary of $\Omega$, and define

$$
g_{*}\left(x_{0}\right)=\liminf _{x \in \omega \rightarrow x_{0}} g(x),
$$

and

$$
g^{*}\left(x_{0}\right)=\limsup _{x \in \omega \rightarrow x_{0}} g(x)
$$

DeFinition 3. The function $\phi(x)$ is an under-function (relative to $g(x))$ in $\Omega$ if $\phi(x)$ is continuous in $\bar{\Omega}$, is $\operatorname{sub}-\{F\}$ in $\Omega$, and $\phi(x) \leqq g(x)$ on $\omega$.

DeFinition 4. The function $\psi(x)$ is an over-function (relative to $g(x))$ in $\Omega$ if $\psi(x)$ is continuous in $\bar{\Omega}$, is a super- $\{F\}$ function in $\Omega$, and $\psi(x) \geqq g(x)$ on $\omega$.

Postulate 4 . If $\Omega$ is any bounded domain comprised together with its boundary $\omega$ in $D$ and if $g(x)$ is any bounded function defined on $\omega$, then the associated families of over-functions and under-functions are both non-null.

Definition 5. By a solution of the Dirichlet Problem for $\Omega$ relative to $\{F(x)\}$ and relative to a given bounded boundary value function $g(x)$ on $\omega$, we shall mean a function $H(x)$ which is continuous in $\Omega$, satisnies

$$
g_{*}\left(x_{0}\right) \leqq \liminf _{x \in \bar{\Omega} \rightarrow x_{0}} H(x) \leqq \lim _{x \in \bar{\Omega} \rightarrow x_{0}} \sup _{1} H(x) \leqq g^{*}\left(x_{0}\right)
$$

at each $x_{0} \in \omega$, and is such that for each $\kappa \in\{\kappa\}$ with $\bar{K} \subset \Omega$ we have

$$
H(x) \equiv F(x ; H ; \kappa) \text { in } \bar{K} .
$$

DeFinition 6. We shall say that a function $H(x)$ which is continuous in $\Omega$, and which satisfies (5) for each $\kappa \in\{\kappa\}$ with $\bar{K} \subset \Omega$, is an $\{F\}$ function in $\Omega$.

Definition 7. Given a bounded domain $\Omega$ such that $\bar{\Omega} \subset D$ and a bounded function $g(x)$ defined on $\omega$. We denote by $H_{*}(x)$ and $H^{*}(x)$ the functions defined by 


$$
H_{*}(x)=\sup _{\phi \in\{\phi\}} \phi(x),
$$

and

$$
H^{*}(x)=\inf _{\varphi \in\{\psi\}} \psi(x),
$$

where $\{\phi\}$ and $\{\psi\}$ are the associated families of under-functions and over-functions respectively.

THEOREM 1. Given any bounded domain $\Omega$ with $\bar{\Omega} \subset D$ and any bounded function $g(x)$ defined on $\omega$, then the associated functions $H_{*}(x)$ and $H^{*}(x)$ are $\{F\}$-functions in $\Omega[1 ;$ p. 303].

DeFinition 8. The point $x_{0} \in \omega$ is a regular boundary point of $\Omega$ relative to $\{F(x)\}$ provided that for every bounded function $g(x)$ defined on $\omega$ the associated functions $H_{*}(x)$ and $H^{*}(x)$ satisfy (4) at $x_{0}$.

THEOREM 2. If all points of $\omega$ are regular boundary points of $\Omega$, and $g(x)$ is continuous on $\omega$, then the Dirichlet problem for $\Omega$, relative to $\{F(x)\}$ and $g(x)$, has a unique solution $[1 ; \mathrm{p}$. 304].

The next theorem shows that regularity of a boundary point "in the small" implies regularity "in the large".

Definition 9. For a point $x_{0} \in \omega$, a circle $\kappa$ with center at $x_{0}$ and with $\bar{K} \subset D$, and constants $\varepsilon>0, M$, and $N$, a function

$$
s(x) \equiv s(x ; \kappa ; \varepsilon, M, N)
$$

is a barrier subfunction provided :

(a) $s(x)$ is continuous in $\bar{\Omega} \cap \bar{K}$,

(b) $s(x)$ is a sub- $\{F\}$ function in $\Omega \cap K$,

(c) $s\left(x_{0}\right) \geqq N-\varepsilon$,

(d) $s(x) \leqq N+2 \varepsilon$ on $\omega \cap \bar{K}$,

and
(e) $s(x) \leqq M$
on $\bar{\Omega} \cap \kappa$.

Definition 10. With the notation of Definition 9, a function $S(x) \equiv$ $S(x ; \kappa ; \varepsilon, M, N)$ is a barrier superfunction provided :

(a) $S(x)$ is continuous in $\bar{K} \cap \bar{\Omega}$,

(b) $S(x)$ is a super- $\{F\}$ function in $K \cap \Omega$,

(c) $S\left(x_{0}\right) \leqq N+\varepsilon$,

(d) $S(x) \geqq N-2 \varepsilon$ on $\omega \cap \bar{K}$,

and
(e) $S(x) \geqq M$
on $\bar{\Omega} \cap \kappa$.

THEOREM 3. If for $x_{0} \in \omega$ and for each set of constants $\varepsilon>0, M$, and $N$, there exists a sequence of circles $\kappa_{n}=\kappa_{n}\left(x_{0}\right)$ with centers at $x_{0}$ and 
radii $r_{n}\left(x_{0}\right) \rightarrow 0$ for which barrier subfunctions $s\left(x ; \kappa_{n} ; \varepsilon, M, N\right)$ and barrier superfunctions $S\left(x ; \kappa_{n} ; \varepsilon, M, N\right)$ exist, then $x_{0}$ is a regular boundary point of $\Omega$ relative to $\{F(x)\}[1 ; \mathrm{p} .305]$.

3. Equicontinuity at the boundary. In this section, before turning our attention to differential equations, we shall show that a property of $\{F\}$-functions given as Postulate 8 in [1] is a consequence of Postulates 1 and 2.

Theorem 4. For any circle $\kappa \in\left\{\kappa_{\}}^{\prime}\right.$, if the functions $\left\{h_{i}(x)\right\}$, uniformly bounded and continuous on $\kappa$, are equicontinuous at $x_{0} \in \kappa$, then the functions $F\left(x ; h_{\nu} ; \kappa\right)$, defined in $\bar{K}$, are equicontinuous at $x_{0}$.

Proof. Assume that $\left|h_{\nu}(x)\right| \leqq M$ on $\kappa$ for all $h_{\nu}(x) \in\left\{h_{\nu}(x)\right\}$. Since the functions $\left\{h_{\nu}(x)\right\}$ are equicontinuous at $x_{0}$, it follows that given $\varepsilon>0$ there exists an arc $\sigma$ of $k$ with midpoint at $x_{0}$ such that

$$
\left|h_{\nu}(x)-h_{\nu}\left(x_{0}\right)\right|<\varepsilon \quad \text { on } \sigma
$$

for all $h_{\nu}(x) \in\left\{h_{\nu}(x)\right\}$. Now let the function $g(x)$ be continuous on $\kappa$, $g(x)>M$ on $\kappa-\sigma, g(x) \geqq-M+\varepsilon$ on $\sigma$, and $g\left(x_{0}\right) \leqq-M+2 \varepsilon$. For any $h_{\nu}(x) \in\left\{h_{\nu}(x)\right\}$ set

$$
c_{\nu}=h_{\nu}\left(x_{0}\right)+M \geqq 0,
$$

then

$$
F\left(x ; h_{\nu} ; \kappa\right)-c_{\nu}<F(x ; g ; \kappa)
$$

on $\kappa$. Therefore, by Postulate 2

$$
F\left(x ; h_{\nu} ; \kappa\right)-c_{\nu}<F(x ; g ; \kappa) \quad \text { in } \bar{K}
$$

for each $h_{\nu}(x) \in\left\{h_{\nu}(x)\right\}$. Since $F(x ; g ; \kappa)$ is continuous in $\bar{K}$, there exists a circle $\kappa_{1}$ with center at $x_{0}$ such that

$$
F(x ; g ; \kappa)-F\left(x_{0} ; g ; \kappa\right)<\varepsilon \quad \text { in } \bar{K} \cap \bar{K}_{1} .
$$

Then

$$
F\left(x ; h_{\nu} ; \kappa\right)-c_{\nu}<F(x ; g ; \kappa)<\varepsilon+F\left(x_{0} ; g ; \kappa\right) \leqq 3 \varepsilon+F\left(x_{0} ; h_{\nu} ; \kappa\right)-c_{\nu}
$$

in $\bar{K} \cap \bar{K}_{1}$, hence, for any $h_{\nu}(x) \in\left\{h_{\nu}(x)\right\}$

$$
F\left(x ; h_{\nu} ; \kappa\right)-F\left(x_{0} ; h_{\nu} ; \kappa\right)<3 \varepsilon \quad \text { in } \bar{K} \cap \bar{K}_{1} .
$$

By a similar argument there exists a circle $\kappa_{2}$ with center $x_{0}$ such that

$$
F\left(x ; h_{\nu} ; \kappa\right)-F\left(x_{0} ; h_{\nu} ; \kappa\right)>-3 \varepsilon \quad \text { in } \bar{K} \cap \bar{K}_{2} .
$$

Hence, if $\kappa_{3}$ is the smaller of $\kappa_{1}$ and $\kappa_{2}$, then 


$$
\left|F\left(x ; h_{\nu} ; \kappa\right)-F\left(x_{0} ; h_{\nu} ; \kappa\right)\right|<3 \varepsilon \quad \text { in } \bar{K} \cap \bar{K}_{3}
$$

and the functions $F\left(x ; h_{\nu} ; \kappa\right)$ are equicontinuous at $x_{0}$.

Theorem 4 obviously remains valid under weaker conditions. For example, the theorem remains valid if Postulate 1 is weakened by assuming that the boundary value problem is solvable for some class of continuous boundary value functions defined on $\kappa$ which under the uniform topology is dense in the set of all continuous functions defined on $\kappa$. Also, Theorem 4 remains valid if instead of dealing with a circle $\kappa \in\{\kappa\}$ we state the theorem in terms of a bounded demain $\Omega$ with $\bar{\Omega} \subset D$ and assume $x_{0}$ is a regular boundary point of $\Omega$. However, in this case the proof draws on Postulates 3 and 4 as well as Postulates 1 and 2 .

4. Applications to elliptic partial differential equations. In this section we shall show that the solutions of certain types of elliptic partial differential equations satisfy Postulates 1 to 4 . We shall also consider some regularity criteria for boundary points with respect to these equations. It will be more convenient in this section to return to the customary $(x, y)$ representation of points in the plane.

First we shall consider Postulate 2 since it states a characteristic property of the solutions of a wide class of elliptic differential equations. We consider the function $E(x, y, z, p, q, r, s, t)$ and make the following assumptions :

(1) $E$ is continuous in all 8 variables in the region $T$ defined by

$$
T:\left\{\begin{array}{l}
(x, y) \in D \\
-\infty<z, p, q, r, s, t<+\infty
\end{array}\right.
$$

where $D$ is a domain in the $x y$-plane.

(2) The first partial derivatives $E_{z}, E_{p}, E_{q}, E_{r}, E_{s}$, and $E_{t}$ are continuous in $T, E_{s}^{2}-4 E_{r} E_{i}<0, E_{r}>0$, and $E_{z} \leqq 0$ in $T$.

THEOREM 5. The solutions of the elliptic partial differential equation

$$
E(x, y, z, p, q, r, s, t)=0
$$

where $p=\frac{\partial z}{\partial x}, q=\frac{\partial z}{\partial y}, r=\frac{\partial^{2} z}{\partial x^{2}}, s=\frac{\partial^{2} z}{\partial x \partial y}$, and $t=\frac{\partial^{2} z}{\partial y^{2}}$ satisfy Postulate 2.

Theorem 6. The functions $s(x, y)$ and $S(x, y)$ of class $C^{(2)}$ in the subdomain $\Omega \subset D$, are respectively a subfunction and a superfunction in $\Omega$ with respect to solutions of (6) if and only if

$$
E\left(x, y, s, s_{x}, s_{y}, s_{x x}, s_{x y}, s_{y y}\right) \geqq 0
$$

and 


$$
E\left(x, y, S, S_{x}, S_{y}, S_{x x}, S_{x y}, S_{y y}\right) \leqq 0
$$

in $\Omega$.

The proofs of Theorems 5 and 6 follow immediately from the maximum principle for solutions of elliptic partial differential equations which has been discussed by Hopf [4].

We consider now the linear elliptic equation

$$
L(z) \equiv \Delta z+a(x, y) z_{x}+b(x, y) z_{y}+c(x, y) z=f(x, y) .
$$

We assume that $D$ is a bounded plane domain such that $a(x, y), b(x, y)$, $c(x, y)$ and $f(x, y)$ are Hölder continuous in $\bar{D}$ and $c(x, y) \leqq 0$ in $\bar{D}$.

\section{Theorem 7. The solutions of (1) satisfy Postulates 1, 2, 3, and 4.}

Proof. It follows from Theorem 5 that the condition $c(x, y) \leqq 0$ in $\bar{D}$ insures that Postulate 2 is satisfied.

It is known [9] that there is an $r_{0}>0$, depending on $\max [|a|,|b|$, $|c|,|f|]$ in $\bar{D}$, such that Postulate 1 is satisfied for the family $\{k\}$ of circles with radii less than or equal to $r_{0}$ and with $\bar{K}=K+\kappa \subset D$. The uniqueness part of Postulate 1 follows since Postulate 2 is satisfied.

If $\kappa \in\{\kappa\}$, if $\left(x_{0}, y_{0}\right)$ is an interior point of $K$, and if $z(x, y)$ is continuous in $\bar{K}$, is of class $C^{(2)}$ in $\bar{K}$, and is a solution of (1) in $K$, then $\left|z_{x}\left(x_{0}, y_{0}\right)\right| \leqq M$ and $\left|z_{y}\left(x_{0}, y_{0}\right)\right| \leqq M$, where $M$ depends on $\max [|a|,|b|,|c|$, $|f|]$ in $\bar{K}, \max |z(x, y)|$ on $\kappa$, the radius of $\kappa$, and the distance from $\left(x_{0}, y_{0}\right)$ to $\kappa$ [9]. This implies that Postulate 3 is satisfied.

Let $\Omega$ be any domain such that $\bar{\Omega} \subset D$ and let $g(x, y)$ be any bounded function defined on $\omega$. Then, if $u(x, y)=\gamma\left[\alpha-e^{\beta x}\right]$ where $\alpha, \beta$, and $\gamma$ are constants,

$$
L[u]=\gamma\left(\alpha-e^{\beta x}\right)\left[c(x, y)-\frac{\left(\beta^{2}+\beta a(x, y) e^{\beta x}\right.}{\alpha-e^{\beta x}}\right] .
$$

Choose $\beta$ so that $\beta>\max |\alpha(x, y)|$ in $\bar{D}$, then choose $\alpha$ so that $\alpha-e^{\beta x}>1$ in $\bar{\Omega}$. It is then clear that $\gamma_{1}>0$ can be chosen large enough that the function $\psi(x, y)=\gamma_{1}\left[\alpha-e^{\beta x}\right]$ will simultaneously satisfy the conditions :

$$
L[\psi] \leqq f(x, y) \text { in } \Omega \text { and } \psi(x, y) \geqq g(x, y) \text { on } \omega .
$$

Hence, it follows from Definition 4 and Theorem 6 that $\psi(x, y)$ is an over-function. Similarly, if $\gamma_{2}>0$ is taken large enough,

$$
\phi(x, y)=-\gamma_{2}\left[\alpha-e^{\beta x}\right]
$$

will be an under-function. Postulate 4 is satisfied. 
Since Postulates 1 to 4 are satisfied it follows from Theorem 1 that, given any domain $\Omega$ with $\bar{\Omega} \subset D$ and any bounded function $g(x, y)$ defined on $\omega$, the associated functions $H^{*}(x, y)$ and $H_{*}(x, y)$ exists and are solutions of (1) in $\Omega$.

TheOREM 8. Let $\Omega$ be a domain with $\bar{\Omega} \subset D$ and let $\left(x_{0}, y_{0}\right) \in \omega$ be such that a circle $\kappa_{0}$ can be drawn with $\bar{K}_{0} \subset \bar{D}$ and $\bar{K}_{0} \cap \bar{\Omega}=\left(x_{0}, y_{0}\right)$. Then $\left(x_{0}, y_{0}\right)$ is a regular boundary point of $\Omega$ relative to solutions of (1)

Proof. Making use of Theorem 3 we see that to establish the regularity of $\left(x_{0}, y_{0}\right)$ it is sufficient to show that barrier subfunctions and barrier superfunctions can be constructed for all sufficiently small circles with centers at $\left(x_{0}, y_{0}\right)$. We shall consider only the barrier superfunctions since the barrier subfunctions can be dealt with in an exactly parallel way.

By the method used in Theorem 7 we can select a functions $S_{0}(x, y)$ which is continuous in $\bar{\Omega}$, is of class $C^{(2)}$ in $\Omega$, and satisfies

$$
L\left[S_{0}\right] \leqq f(x, y) \quad \text { in } \Omega \text {. }
$$

Now assume that constants $\varepsilon>0, M$, and $N$ are given. Let $\left(x_{1}, y_{1}\right)$ be the center of $\kappa_{0}$ and $r_{0}$ its radius. Let $\kappa_{1}$ be a circle with center at $\left(x_{0}, y_{0}\right)$ and radius $r_{1}<r_{0}$ taken small enough that

$$
S_{0}(x, y) \geqq S_{0}\left(x_{0}, y_{0}\right)-\varepsilon \quad \text { on } \omega \cap \bar{K}_{1} .
$$

Let

$$
r=\sqrt{\left(x-x_{1}\right)^{2}+\left(y-y_{1}\right)^{2}}
$$

and

$$
w(x, y)=r_{0}^{-n}-r^{-n} .
$$

One can easily verify that, if $n$ is chosen large enough, then

$$
L[w] \leqq 0 \quad \text { in } \Omega \text {, }
$$

furthermore, $w(x, y)$ is continuous in $\bar{\Omega}, w\left(x_{0}, y_{0}\right)=0$, and $w(x, y)>0$ elsewhere in $\bar{\Omega}$.

Now we consider two cases: $N-S_{0}\left(x_{0}, y_{0}\right) \geqq 0$ and $N-S_{0}\left(x_{0}, y_{0}\right)<0$. First we assume $N-S_{0}\left(x_{0}, y_{0}\right) \geqq 0$, then we can choose $h_{1}>0$ such that

$$
h_{1} w(x, y) \geqq M+\max _{(x, y) \in \Omega}\left|S_{0}(x, y)\right| \quad \text { on } \kappa_{1} \cap \bar{\Omega} .
$$

The function

$$
S\left(x, y ; \kappa_{1} ; \varepsilon, M, N\right)=h_{1} w(x, y)+S_{0}(x, y)+N-S_{0}\left(x_{0}, y_{0}\right)
$$


is then a barrier superfunction at $\left(x_{0}, y_{0}\right)$ for the circle $\kappa_{1}$. This follows immediately from (9), (10), (11), (12) and the definition of a barrier superfunction.

Now assume $N-S_{0}\left(x_{0}, y_{0}\right)<0$. Again it is easily verified that, for $\delta>0$ chosen sufficiently large, the function

$$
v(x, y)=\left[N-S_{0}\left(x_{0}, y_{0}\right)\right] e^{\delta\left(x-x_{0}\right)}
$$

satisfies

$$
L[v] \leqq 0 \quad \text { in } \Omega .
$$

Let the circle $\kappa_{2}$ with center at $\left(x_{0}, y_{0}\right)$ and radius $r_{2}<r_{1}$ be chosen small enough that

$$
v(x, y) \geqq N-S_{0}\left(x_{0}, y_{0}\right)-\quad \text { on } \omega \cap \bar{K}_{2} .
$$

Then let $h_{2}>0$ be taken large enough that

$$
h_{2} w(x, y) \geqq M+\max _{(x, y) \in \bar{\Omega}}\left[\left|S_{0}(x, y)\right|+|v(x, y)|\right] \quad \text { on } \kappa_{2} \cap \bar{\Omega} .
$$

It follows from (13), (14), (15), and (16) that

$$
S\left(x, y ; \kappa_{2} ; \varepsilon, M, N\right)=h_{2} w(x, y)+v(x, y)+S_{0}(x, y)
$$

is a barrier superfunction at $\left(x_{0}, y_{0}\right)$ with respect to the circle $\kappa_{2}$.

Theorem 9. Let $D$ be a domain in which the coefficient functions in (1) are Hölder continuous. Then, if $\Omega$ is any bounded domain with $\bar{\Omega} \subset D$ and is such that corresponding to each $\left(x_{0}, y_{0}\right) \in \omega$ there is a circle $\kappa$ with $\bar{\Omega} \cap \bar{K}=\left(x_{0}, y_{0}\right)$ and if $g(x, y)$ is any continuous function defined on $\omega$, there is a unique function $z(x, y)$ which is continuous in $\bar{\Omega}$, is of class $C^{(2)}$ and satisfies (1) in $\Omega$, and is equal to $g(x, y)$ on $\omega$.

Proof. This is an immediate consequence of Theorems 2 and 8. In our consideration of the quasi-linear equation

$$
a(p, q) r+2 b(p, q) s+c(p, q) t=0
$$

we are going to employ two sets of conditions on the coefficient functions, first, conditions (A): $a(p, q), b(p, q)$, and $c(p, q)$ have Hölder continuous first partial derivatives, $a c-b^{2}=1$, and $a>0$ for all $(p, q)$.

Bers [2] has proved that, if $a, b$, and $c$ satisfy conditions (A), then there exist functions $k(p, q), \theta(p, q)$, and $\Lambda(p, q)$ with $k(p, q)>0, \theta(0,0)$ $=\Lambda(0,0)=0$, and which are such that

$$
\frac{\partial \theta}{\partial p}=k a \quad \frac{\partial \Lambda}{\partial p}+\frac{\partial \theta}{\partial q}=2 k b \quad \frac{\partial \Lambda}{\partial q}=k c .
$$


We now state conditions (B) on the coefficients of (2): There exists an $\varepsilon>0$ such that

$$
a\left(\frac{1+p^{2}}{w}\right)+c\left(\frac{1+q^{2}}{w}\right)+2 b\left(\frac{p q}{w}\right) \leqq 2 \varepsilon
$$

for all $(p, q)$ where $w=\sqrt{1+p^{2}+q^{2}}$, further $\theta$ and $\Lambda$ can be chosen so that

$$
\frac{\theta^{2}+\Lambda^{2}}{p \theta+q \Lambda} \leqq 1
$$

for all $(p, q)$. Conditions (A) and (B) are satisfied by the minimal surface equation (3) if it is normalized so that $a c-b^{2}=1$. For this reason Finn [3] calls equations (2) which satisfy conditions (A) and (B) equations of " minimal surface type".

In our application of the theory of $\S 2$ to equation (2), we let $D$ be the $x y$-plane and $\{k\}$ the family of all circles in the plane.

THEOREM 10. If equation (2) satisfies conditions $(A)$ and $(B)$, then its solutions satisfy Postulates 1 to 4.

Proof. Nirenberg [7; p. 138] has proved that if $\Gamma$ is any convex domain in the plane with boundary $\gamma$ which is of finite length, which can be represented parametrically by

$$
\gamma:\left\{\begin{array}{l}
x=x(s) \\
y=y(s)
\end{array}\right.
$$

in terms of arc length $s$ where $x(s)$ and $y(s)$ are of class $C^{(3)}$, and which has positive curvature everywhere, and if $g(s)$ has a Hölder continuous second derivative on $\gamma$, then there is a function $z(x, y)$ continuous in $\bar{\Gamma}$, of class $C^{(2)}$ and a solution of (2) in $\Gamma$, and such that $z(x(s), y(s))$ $=g(s)$ on $\gamma$.

Finn [3] has shown that if (2) satisfies conditions (A) and (B) and if $z(x, y)$ is continuous in $\bar{K}$, is of class $C^{(3)}$ in $K$, and is a solution of (2) in $K$, then at any point $\left(x_{0}, y_{0}\right) \in K\left|z_{x}\left(x_{v}, y_{0}\right)\right| \leqq M$ and $\left|z .\left(x_{0}, y_{0}\right)\right| \leqq M$ where $M$ depends on $\max |z(x, y)|$ on $\kappa$, the radius of $\kappa$, the distance from $\left(x_{0}, y_{0}\right)$ to $\kappa$, and other quantities which are fixed for any particular equation (2). Using standard arguments [3; p. 411], one can then use Nirenberg's result to prove that Postulate 1 is satisfied. The bounds on the first partial derivatives of solutions imply that Postulate 3 is satisfied. That Postulate 2 is satisfied follows from Theorem 5 and since planes are solutions of (2) Postulate 4 is obviously satisfied.

Thus, we can conclude that, if $\Omega$ is any bounded domain and $g(x, y)$ 
is any bounded function defined on $\omega$, the functions $H^{*}(x, y)$ and $H_{*}(x, y)$ of Theorem 1 exist and are solutions of (2) in $\Omega$. In particular this is true of the minimal surface equation.

THEOREM 11. Let equation (2) satisfy conditions $(A)$ and $(B)$ and let $\Omega$ be any bounded plane domain with boundary $\omega$. If $\left(x_{0}, y_{0}\right) \in \omega$ is such that there is a circle $\kappa$ with center at $\left(x_{0}, y_{0}\right)$ and a straight line $\pi$ such that $\pi \cap(\bar{K} \cap \bar{\Omega})=\left(x_{0}, y_{0}\right)$, then $\left(x_{0}, y_{0}\right)$ is a regular boundary point of $\Omega$ relative to solutions of $(2)$.

Proof. Since planes are solutions of (2), barrier subfunctions and superfunctions can obviously be constructed at $\left(x_{0}, y_{0}\right)$ for all sufficiently small circles with centers at $\left(x_{0}, y_{0}\right)$.

It follows that if equation (2) satisfies conditions (A) and (B), then in order that the Dirichlet problem have a solution for any convex domain whose boundary contains no straight line segments, it is sufficient that the Dirichlet problem have a solution for circles. Of course it is well known that the Dirichlet problem for the minimal surface equation always has a solution for convex domains whether or not their boundaries contain straight line segments. It is known that the Dirichlet problem for equation (2) is not always solvable for non-convex domains. In particular an example of a boundary value problem for a non-convex region which is not solvable for the minimal surface equation was given by H. A. Schwarz [8; p. 42]. For a given domain $\Omega$ with boundary $\omega$, those points of $\omega$ which satisfy the criterion of Theorem 11 are regular with respect to equation (2), those points which are interior points of straight line segments of $\omega$ are possibly regular, but it seems likely that all other points of $\omega$ are not regular relative to solutions of (2). The possibility remains that the Dirichlet problem for (2) for certain types of non-convex domains may be solvable if the boundary values are suitably restricted. Our last theorem contains a weak result in this direction.

Let $\omega_{c}$ be the set of points of $\omega$ which satisfy the regularity criterion of Theorem 11. Let $\omega_{n}=\omega-\omega_{c}$ and for $\delta>0$ let $\omega_{\delta}$ be the set of points of $\omega$ which belong to $\omega_{n}$ or are within a distance $\delta$ of points of $\omega_{n}$.

THEOREM 12. Let $\Omega$ be a bounded plane domain with boundary $\omega$ for which there is an $R>0$ such that for every $(x, y) \in \omega$ a circle $\kappa$ of radius $R$ may be drawn with $\bar{\Omega} \cap \bar{K}=(x, y)$. If for a given $\delta>0$ the boundary value function $g(x, y)$ is continuous on $\omega$, is constant on each component of $\omega_{\delta}$, and is such that 


$$
V_{g} \equiv \max _{(x, y) \in \omega} g(x, y)-\min _{(x, y) \in \omega} g(x, y) \leqq \begin{cases}\frac{\delta}{\sqrt{2}} & \text { for } \delta \leqq R \\ R \sqrt{\frac{\delta}{\delta+R}} & \text { for } \delta>R,\end{cases}
$$

then the Dirichlet problem for $\Omega$ with boundary values $g(x, y)$ has a unique solution for the minimal surface equation (3).

Proof. As we have already observed the functions $H_{*}(x, y)$ and $H^{*}(x, y)$ both exist and are solutions of (3) in $\Omega$. Since the function $g(x, y)$ is continuous on $\omega$, it is sufficient to show that inequality (4) is satisfied at each point of $\omega$. This implies that the functions $H_{*}(x, y)$ and $H^{*}(x, y)$ are both continuous in $\bar{\Omega}$, agree on $\omega$, and consequently coincide in $\bar{\Omega}$ to give the unique solution of the Dirichlet problem. Since by Theorem 11 the points of $\omega_{c}$ are regular, it will be sufficient to show that at each point of $\omega_{n}$ we can construct an over-function and an under-function which take on the given boundary value at the point.

Let $\left(x_{0}, y_{0}\right) \in \omega_{n}$ and let $\kappa_{0}$ be a circle of radius $R$ such that $\bar{K}_{0} \cap \bar{\Omega}$ $=\left(x_{0}, y_{0}\right)$. Translate the origin to the center of $\kappa_{0}$ and rotate the axes so that $\left(x_{0}, y_{0}\right)$ becomes the point $(R, 0)$. Draw the circle $\kappa_{1}$ with center at $(R, 0)$ and radius $\delta$. Then the function

$$
S_{1}(x, y)=\frac{R \sqrt{x^{2}+y^{2}-R x}}{\sqrt{x^{2}+y^{2}}}
$$

is of class $C^{(2)}$ on comp $\bar{K}_{0}, S_{1}(x, y) \geqq 0$ on comp $K_{0}$, and $S_{1}(R, 0)=0$. Furthermore, by substituting $S_{1}(x, y)$ in the left-hand member of equation (3) one can verify that inequality (8) is satisfied in $\Omega$. It follows from Theorem 6 that $S_{1}(x, y)$ is a superfunction in $\Omega$ with respect to solutions of (3). Finally, we also have that on $\kappa_{1} \cap \operatorname{comp} K_{0}$

$$
\min S_{1}(x, y)= \begin{cases}\frac{\delta}{\sqrt{2}} & \text { if } \delta \leqq R \\ R \sqrt{\frac{\delta}{\delta+R}} & \text { if } \delta>R\end{cases}
$$

We define the function $S_{2}(x, y)$ by

$$
S_{2}(x, y)=S_{1}(x, y)+g(R, 0) .
$$

The function $S_{2}(x, y)$ is clearly also a superfunction in $\Omega$ because of the form of equation (3). Now let $M=\max g(x, y)$ on $\omega$, then the function

$$
\psi(x, y)= \begin{cases}M & \text { in } \bar{\Omega} \cap \operatorname{comp} \overline{K_{1}} \\ \min \left[M, S_{2}(x, y)\right] & \text { in } \bar{\Omega} \cap \bar{K}_{1}\end{cases}
$$


is the desired over-function. To see this we first observe that $\psi(x, y)$ is continuous in $\bar{\Omega}$ since by (19), (21), and (22) $S_{2}(x, y) \geqq M$ on $\bar{\Omega} \cap \kappa_{1}$. The argument that $\psi(x, y)$ is a superfunction is the same as that given in $\left[1 ;\right.$ p. 306]. From the definitions of $S_{2}(x, y)$ and $\omega_{\delta}$ it follows that $\psi(R, 0)=g(R, 0)$ and $\psi(x, y) \geqq g(x, y)$ on $\omega$.

Similarly

$$
s_{1}(x, y)=-S_{1}(x, y),
$$

and

$$
s_{2}(x, y)=s_{1}(x, y)+g(R, 0)
$$

are subfunctions in $\Omega$. The function $\phi(x, y)$ defined by

$$
\phi(x, y)= \begin{cases}m & \text { in } \bar{\Omega} \cap \operatorname{comp} \bar{K}_{1} \\ \max \left[m, s_{2}(x, y)\right] & \text { in } \bar{\Omega} \cap \bar{K}_{1}\end{cases}
$$

where $m=\min g(x, y)$ on $\omega$ is an under-function with $\phi(R, 0)=g(R, 0)$. Thus inequality (4) holds at every point of $\omega$ and

$$
H^{*}(x, y) \equiv H_{*}(x, y) \quad \text { in } \bar{\Omega}
$$

constituting the unique solution of the Dirichlet problem.

\section{REFERENCES}

1. E. F. Beckenbach and L. K. Jackson Subfunctions of several variables, Pacific J. Math. 3 (1953), 219-313.

2. L. Bers, Univalent solutions of linear elliptic systems, Comm. Pure Appl. Math. 6 (1953), 513-526.

3. R. Finn, On equations of minimal surface type, Ann. of Math. 60 (1954), 347-416.

4. E. Hopf, Elementare Bemerkungen uber die Lösungen partieller Differentialgleichungen zweiter Ordnung vom elliptischen Typus, Sitzungsber. Preuss. Akad. Wiss. 19 (1927), 147250.

5. M. Inoue, Dirichlet problem relative to a family of functions. J. Inst, Polytech., Osaka City Uni., 7 (1956), 1-16.

6. L. K. Jackson, On generalized subharmonic functions, Pacific J. Math. 5 (1955), 215-228.

7. L. Nirenberg, Cn nonlinear elliptic partial differential equations and Hölder continuity, Comm. Pure Appl. Math. 6 (1953), 103-156.

8. T. Radó, On the problem of Plateau, Chelsea, New York, 1951.

9. J. Schauder, Üter lineare ellipticche Differential-gleichungen zweiter Ordnung, Math.

Z. 38 (1934), 257-282.

UNIVERSITY OF NEBRASKA 



\section{PACIFIC JOURNAL OF MATHEMATICS}

\section{EDITORS}

\section{H. L. Royden}

Stanford University

Stanford, California

\section{R. A. Beaumont}

University of Washington

Seattle 5 , Washington

\author{
A. L. Whiteman
}

University of Southern California

Los Angeles 7, California

E. G. Straus

University of California

Los Angeles 24, California

\section{ASSOCIATE EDITORS}
E. F. BECKENBACH
A. HORN
L. NACHBIN
G. SZEKERES
C. E. BURGESS
V. GANAPATHY IYER
I. NIVEN
F. WOLF
M. HALL
R. D. JAMES
T. G. OSTROM
E. HEWITT
M. S. KNEBELMAN
M. M. SCHIFFER
K. YOSIDA

\section{SUPPORTING INSTITUTIONS}

\author{
UNIVERSITY OF BRITISH COLUMBIA \\ CALIFORNIA INSTITUTE OF TECHNOLOGY \\ UNIVERSITY OF CALIFORNIA \\ MONTANA STATE UNIVERSITY \\ UNIVERSITY OF NEVADA \\ OREGON STATE COLLEGE \\ UNIVERSITY OF OREGON \\ UNIVERSITY OF SOUTHERN CALIFORNIA
}

\author{
STANFORD UNIVERSITY \\ UNIVERSITY OF UTAH \\ WASHINGTON STATE COLLEGE \\ UNIVERSITY OF WASHINGTON \\ * * * * \\ AMERICAN MATHEMATICAL SOCIETY \\ CALIFORNIA RESEARCH CORPORATION \\ HUGHES AIRCRAFT COMPANY \\ THE RAMO-WOOLDRIDGE CORPORATION
}

Mathematical papers intended for publication in the Pacific Journal of Mathematics should be typewritten (double spaced), and the author should keep a complete copy. Manuscripts may be sent to any of the editors. All other communications to the editors should be addressed to the managing editor, E. G. Straus at the University of California, Los Angeles 24, California.

50 reprints per author of each article are furnished free of charge; additional copies may be obtained at cost in multiples of 50 .

The Pacific Journal of Mathematics is published quarterly, in March, June, September, and December. The price per volume (4 numbers) is $\$ 12.00$; single issues, $\$ 3.50$. Back numbers are available. Special price to individual faculty members of supporting institutions and to individual members of the American Mathematical Society: $\$ 4.00$ per volume; single issues, $\$ 1.25$.

Subscriptions, orders for back numbers, and changes of address should be sent to Pacific Journal of Mathematics, 2120 Oxford Street, Berkeley 4, California.

Printed at Kokusai Bunken Insatsusha (International Academic Printing Co., I.td.), No. 10, 1-chome, Fujimi-cho, Chiyoda-ku, Tokyo, Japan.

PUBLISHED BY PACIFIC JOURNAL OF MATHEMATICS, A NON-PROFIT CORPORATION

The Supporting Institutions listed above contribute to the cost of publication of this Journal, but they are not owners or publishers and have no responsibility for its content or policies. 


\section{Pacific Journal of Mathematics}

\section{Vol. 8, No. 2 \\ April, 1958}

John Herbert Barrett, Second order complex differential equations with a real independent variable ............................ 187

Avner Friedman, Remarks on the maximum principle for parabolic equations and its applications ......................... 201

Richard Robinson Goldberg, An inversion of the Stieltjes transform ....... 213

Olavi Hellman, On the periodicity of the solution of a certain nonlinear integral equation .................................. 219

Gilbert Helmberg, A theorem on equidistribution on compact groups...... 227

Lloyd Kenneth Jackson, Subfunctions and the Dirichlet problem ......... 243

Naoki Kimura, The structure of idempotent semigroups. I ............ 257

Stephen Kulik, A method of approximating the complex roots of equations........................................ 277

Ancel Clyde Mewborn, A note on a paper of L. Guttman.............. 283

Zeev Nehari, On the principal frequency of a membrane ............ 285

G. Pólya and I. J. Schoenberg, Remarks on de la Vallée Poussin means and convex conformal maps of the circle ...................... 295

B. M. Stewart, Asymmetry of a plane convex set with respect to its centroid .......................................... 335

Hans F. Weinberger, Lower bounds for higher eigenvalues by finite difference methods

Edwin Weiss and Neal Zierler, Locally compact division rings ......... 369

Bertram Yood, Homomorphisms on normed algebras ................. 373 